\title{
Biscalar Integrable Conformal Field Theories in Any Dimension
}

\author{
Vladimir Kazakov ${ }^{1,2}$ and Enrico Olivucci ${ }^{3}$ \\ ${ }^{1}$ Laboratoire de Physique Théorique de l'École Normale Supérieure, 24 rue Lhomond, F-75231 Paris Cedex 05, France \\ ${ }^{2}$ PSL University, Sorbonne Universités, UPMC Univ. Paris 06, CNRS, 75005 Paris, France \\ ${ }^{3}$ II. Institut für Theoretische Physik, Universität Hamburg, Luruper Chaussee 149, 22761 Hamburg, Germany
}

(Received 21 May 2018; revised manuscript received 23 July 2018; published 25 September 2018)

\begin{abstract}
We propose a $D$-dimensional generalization of 4D biscalar conformal quantum field theory recently introduced by Gürdogan and one of the authors as a particular strong-twist limit of $\gamma$-deformed $N=4$ supersymmetric Yang-Mills theory. Similar to the 4D case, the planar correlators of this $D$-dimensional theory are conformal and dominated by "fishnet" Feynman graphs. The dynamics of these graphs is described by the integrable conformal $S O(1, D+1)$ spin chain. In $2 \mathrm{D}$, it is the analogue of Lipatov's $S L(2, C)$ spin chain for the Regge limit of QCD but with the spins $s=1 / 4$ instead of $s=0$. Generalizing recent 4D results of Grabner, Gromov, Korchemsky, and one of the authors to any $D$, we compute exactly at any coupling a four-point correlation function dominated by the simplest fishnet graphs of cylindric topology and extract from it exact dimensions of operators with chiral charge 2 and any spin together with some of their operator product expansion structure constants.
\end{abstract}

DOI: 10.1103/PhysRevLett.121.131601

Introduction.-Conformal field theories (CFTs) are ubiquitous in two dimensions [1], and quite a few supersymmetric CFTs in $D=3,4,6$ dimensions are known. But well-defined nonsupersymmetric CFTs in $D>2$, such as 3D Ising or Potts models or the Banks-Zaks model [2], are rare species despite their rich potential applications ranging from the theory of phase transitions to fundamental interactions. The CFTs at $D>2$ which in addition are integrable, such as $4 \mathrm{D} N=4$ supersymmetric Yang-Mills (SYM) and Aharony-Bergman-Jafferis-Maldacena (ABJM) theories in 't Hooft limit, are true exceptions [3,4]. That is why a new family of planar integrable CFTs obtained in Ref. [5] as a special double-scaling limit of $\gamma$-deformed $N=4$ SYM theory seems to be an important and instructive example. This theory can be studied via quantum spectral curve (QSC) formalism [6-8] or using the integrability of its dominant Feynman graphs via the conformal $S U(2,2)$ noncompact spin chain. A nice particular case of this family is the $4 \mathrm{D}$ biscalar theory, whose planar limit is dominated by "fishnettype" Feynman graphs [5,9].

We propose here the following $D$-dimensional generalization of the 4D biscalar theory introduced in Ref. [5]

$$
\begin{aligned}
\mathcal{L}_{\phi}= & N_{c} \operatorname{tr}\left[\phi_{1}^{\dagger}\left(-\partial_{\mu} \partial^{\mu}\right)^{\omega} \phi_{1}+\phi_{2}^{\dagger}\left(-\partial_{\mu} \partial^{\mu}\right)^{(D / 2)-\omega} \phi_{2}\right. \\
& \left.+(4 \pi)^{(D / 2)} \xi^{2} \phi_{1}^{\dagger} \phi_{2}^{\dagger} \phi_{1} \phi_{2}\right],
\end{aligned}
$$

Published by the American Physical Society under the terms of the Creative Commons Attribution 4.0 International license. Further distribution of this work must maintain attribution to the author(s) and the published article's title, journal citation, and DOI. Funded by SCOAP . where both scalar fields transform under the adjoint representation of $S U\left(N_{c}\right), \xi^{2}$ is the coupling constant, and $\omega \in[0,(D / 2)]$ is a deformation parameter. The nonlocal (for general $D, \omega$ ) operators in kinetic terms should be understood as an integral kernel

$$
\left(\partial_{\mu} \partial^{\mu}\right)^{\beta} f(x) \equiv \frac{(-4)^{\beta} \Gamma\left(\frac{D}{2}+\beta\right)}{\pi^{\frac{D}{2}} \Gamma(-\beta)} \int \frac{d^{D} y f(y)}{|x-y|^{D+2 \beta}} .
$$

The propagator of scalar fields is its functional inverse:

$$
\begin{aligned}
\left(-\partial_{\mu} \partial^{\mu}\right)^{\beta} D(x) & =\delta^{(D)}(x), \\
D(x-y) & =\frac{\Gamma\left(\frac{D}{2}-\beta\right)}{4^{\beta} \pi^{\frac{D}{2}} \Gamma(\beta)|x-y|^{D-2 \beta}} .
\end{aligned}
$$

The typical structure in the bulk of sufficiently big planar Feynman graphs in this theory is that of the regular square lattice (fishnet graphs proposed in Ref. [10] as an integrable lattice spin model) by the same reasons as in the 4D case $[5,9]$, namely, due to the presence of the single chiral interaction vertex in the Lagrangian and the absence of its Hermitian conjugate. For example, the graphs renormalizing local "vacuum" operator $\operatorname{tr}\left(\phi_{j}\right)^{L}$ are those of the "wheel" type, and they can be studied via the integrable conformal $S O(2, D)$ spin chain [11], as was suggested for the 4D case in Ref. [12]. The dimensions of operators of the type $\operatorname{tr}\left[\phi_{1}^{3}\left(\phi_{2}^{\dagger} \phi_{2}\right)^{k}\right]$ have been also studied in 4D [12] by QSC methods. It is not clear whether this method can be generalized to our $D$-dimensional model, but the spin chain methods certainly can. 
In general, the propagators of the fishnet graphs of the model (1) are different in two different directions: $|x-y|^{-D+2 \omega}$ for $\phi_{1}$ fields and $|x-y|^{-2 \omega}$ for $\phi_{2}$ fields. Let us concentrate here on the "isotropic" case $\omega=D / 4$. In order to maintain the renormalizability, we should add to Eq. (1) the following double-trace counterterms [13,14]:

$$
\begin{aligned}
\mathcal{L}_{\mathrm{DT}} /(4 \pi)^{\frac{D}{2}}= & \alpha_{1}^{2} \sum_{i=1}^{2} \operatorname{tr}\left(\phi_{i} \phi_{i}\right) \operatorname{tr}\left(\phi_{i}^{\dagger} \phi_{i}^{\dagger}\right)-\alpha_{2}^{2} \operatorname{tr}\left(\phi_{1} \phi_{2}\right) \operatorname{tr}\left(\phi_{2}^{\dagger} \phi_{1}^{\dagger}\right) \\
& -\alpha_{2}^{2} \operatorname{tr}\left(\phi_{1} \phi_{2}^{\dagger}\right) \operatorname{tr}\left(\phi_{2} \phi_{1}^{\dagger}\right) .
\end{aligned}
$$

Notice that the first term disappears in the "nonisotropic" case $\omega \neq(D / 4)$ since the couplings of two terms in the first line of Eq. (4) would become dimensionful.

As it was suggested in Ref. [15] and explicitly shown in Ref. [16] for the 4D case, the isotropic biscalar theory with Lagrangian $\mathcal{L}_{\phi}+\mathcal{L}_{\mathrm{DT}}$ has two fixed points. We generalize here this result to any dimension, up to two loops, computing the corresponding Feynman graphs (Fig. 1) contributing to the $\beta_{\alpha_{1}}$ function. Its two zeroes are

$$
\alpha_{1}^{2}(\xi)=\mp \frac{i \xi^{2}}{2}-J^{(D)} \xi^{4}+O\left(\xi^{6}\right),
$$

where the real coefficient $J^{(D)}$ depends on the $\epsilon^{-1}$ coefficient of the lower-left graph of Fig. 1 in dimensional regularization. For example, $J^{(4)}=1 / 2, \quad J^{(2)}=2 \ln 2$, $J^{(1)}=(\pi+4 \ln 2) /(2 \sqrt{\pi})$ [17]. At this critical coupling $\alpha_{1}(\xi)$ the biscalar theory becomes a genuine nonunitary CFT at any coupling $\xi$. The operators $\operatorname{tr}\left(\phi_{1} \phi_{2}\right)$ and $\operatorname{tr}\left(\phi_{1} \phi_{2}^{\dagger}\right)$ are protected in the planar limit as in Ref. [16].

In this Letter, generalizing the 4D results of Ref. [16] to any $D$, we will compute exactly a particular four-point function and read off from it the exact scaling dimensions and certain OPE structure constants of operators of the type $\operatorname{tr}\left[\phi_{1} \partial_{+}^{S} \phi_{1}\left(\phi_{2}^{\dagger} \phi_{2}\right)^{k}\right]+$ permutations. Their dimensions will be given by a remarkably simple exact relation

$$
h_{\Delta, S} \equiv \frac{\Gamma\left(\frac{3 D}{4}-\frac{\Delta-S}{2}\right)}{\Gamma\left(\frac{D}{4}-\frac{\Delta-S}{2}\right)} \frac{\Gamma\left(\frac{D}{4}+\frac{\Delta+S}{2}\right)}{\Gamma\left(-\frac{D}{4}+\frac{\Delta+S}{2}\right)}=\xi^{4},
$$

which reduces of course at $4 \mathrm{D}$ to the result of Ref. [16]. For even $D$, it gives $D$ different solutions $\Delta(\xi)=\Delta_{0}+\gamma(\xi)$.

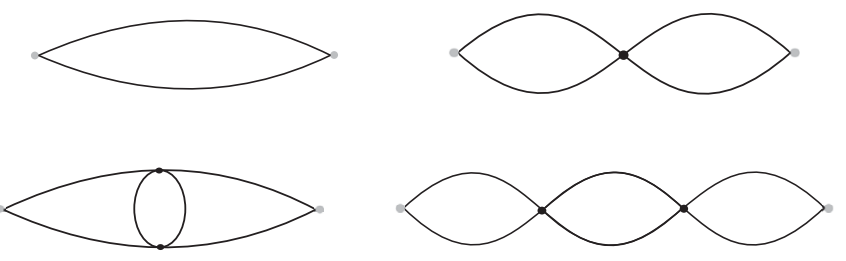

FIG. 1. Loop expansion of $\left\langle\operatorname{tr}\left(\phi_{1}^{2}\right)(x) \operatorname{tr}\left(\phi_{1}^{2}\right)^{\dagger}(0)\right\rangle$ planar graphs up to two loops.
At odd (or noninteger) $D$, there are infinitely many, in general complex, solutions. At weak coupling, the two complex conjugate solutions at $S=0$ [19]

$$
\gamma= \pm i \frac{2 \xi^{2}}{\Gamma\left(\frac{D}{2}\right)} \pm \frac{i}{6} \frac{\xi^{6}}{\Gamma\left(\frac{D}{2}\right)^{3}}\left[\pi^{2}-6 \psi^{(1)}\left(\frac{D}{2}\right)\right]+O\left(\xi^{10}\right)
$$

describe anomalous dimensions of the operator $\operatorname{tr}\left(\phi_{1} \phi_{1}\right)$ at the two fixed points. In a similar way, for any $S \in 2 Z$, the real weak coupling solution

$$
\begin{aligned}
\gamma= & -2 \frac{\xi^{4} \Gamma(S)}{\Gamma\left(\frac{D}{2}\right) \Gamma\left(\frac{D}{2}+S\right)}+\frac{2 \xi^{8} \Gamma(S)^{2}}{\Gamma\left(\frac{D}{2}\right)^{2} \Gamma\left(\frac{D}{2}+S\right)^{2}} \\
& \times\left[\psi^{(0)}\left(\frac{D}{2}\right)-\psi^{(0)}\left(\frac{D}{2}+S\right)+\psi^{(0)}(S)+\gamma_{E}\right] \\
& +O\left(\xi^{10}\right)
\end{aligned}
$$

describes the operators of the type $\operatorname{tr}\left(\phi_{1} \partial_{+}^{S} \phi_{1}\right)$, where $\partial_{+}^{S}=(\hat{n} \cdot \partial)^{S}$ with $\hat{n}$ being an auxiliary lightlike vector. For $D=2 m, m \in \mathbb{N}$ the lhs of Eq. (6) factorizes into a polynomial of degree $2 m$, and $2 m$ roots of Eq. (6) describe the scaling dimension of the exchanged operators in the OPE channel $x_{3} \rightarrow x_{4}$ of Eq. (11) together with their shadows $\tilde{\Delta}=D-\Delta$. At $\xi=0$, we get for the bare dimensions of physical operators (i.e., excluding "shadow" operators)

$$
\Delta_{0}-S=\{m, m+2, \ldots, 3 m-2\} .
$$

At $D=2$, there is a single solution with the dimension $\Delta=1+\sqrt{S^{2}-4 \xi^{4}}$ of the local twist-2 operators $\operatorname{tr}\left(\phi_{1} \partial_{+}^{S} \phi_{1}\right)$, while at 4D the additional $\Delta_{0}-S=4$ describes twist-4 operators [16].As an example, at $D=6$ and $S=0$ the possible nonshadow solutions for Eq. (6) are $\Delta_{0}=3,5,7$. They can be realized as $\operatorname{tr}\left(\phi_{1}^{2}\right)$ for $\Delta_{0}=3$, linear combinations of $\operatorname{tr}\left(\phi_{1} \Delta \phi_{1}\right), \operatorname{tr}\left(\partial_{\mu} \phi_{1} \partial^{\mu} \phi_{1}\right)$ for $\Delta_{0}=5, \quad$ and of $\operatorname{tr}\left(\phi_{1} \Delta^{2} \phi_{1}\right), \operatorname{tr}\left(\Delta \phi_{1} \Delta \phi_{1}\right)$, $\operatorname{tr}\left(\partial_{\mu} \phi_{1} \partial^{\mu} \Delta^{2} \phi_{1}\right), \operatorname{tr}\left(\partial_{\mu} \partial_{\nu} \phi_{1} \partial^{\mu} \partial^{\nu} \phi_{1}\right)$ for $\Delta_{0}=7$.

Diagonalizing the mixing matrix of these operators at $\xi \neq 0$, we would obtain operators with nontrivial, $\xi$ dependent anomalous dimensions, as well as the so-called log-multiplets $[12,20]$ omnipresent in this nonunitary theory $[21,22]$, containing the operators with zero anomalous dimension. Equation (6) predicts that all the exchange operators from this set acquire nontrivial anomalous dimensions, whereas the operators belonging to logmultiplets never appear among them. This appears to be true at any even dimension $D$.

As a general rule, according to Eq. (6), the operators of the type $\left\{\operatorname{tr}\left[\phi_{1}^{2} \partial_{+}^{S}\left(\phi_{2} \phi_{2}^{\dagger}\right)^{k}\right]+\right.$ permutations $\}$ appear in the multiplets only at $D / 4 \in \mathbb{N}, k=1$. We will find below from the exact four-point function the conformal structure constants of these operators with two scalar fields. 


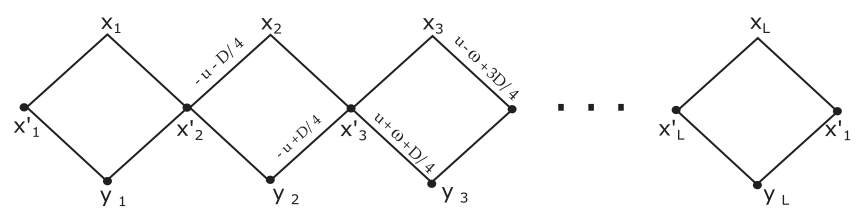

FIG. 2. Graphical representation of the transfer matrix as a convolution of $R$ kernels according to formulas (8) and (9). Black dots are integration points, and the weights of propagators are written in the second and third $R$ kernel.

Integrability of D-dimensional biscalar CFT.-As it was noticed in Ref. [5] and further developed in Refs. [9,12,16], the $4 \mathrm{D}$ case of the theory (1) with $\omega=1$ is integrable in the planar limit. On the one hand, this integrability is the direct consequence of integrability of $\gamma$-twisted planar $N=4$ SYM theory, from which it was obtained in the doublescaling limit combining strong imaginary twist and weak coupling. On the other hand, this integrability was explicitly related in Refs. [5,12] to the fact that the biscalar theory was dominated by the integrable fishnet Feynman graphs [10,23].

Apart from the 4D case, at arbitrary $D$ our biscalar model (1) does not have any integrable SYM origin. But the arguments of equivalence to the integrable conformal $S O(1, D+1)$ spin chain do work. Namely, let us introduce the $D$-dimensional analogue of the 4D "graph-building" operator [5] at general $\omega$ deformation

$$
\begin{aligned}
\mathcal{H}_{L} & \Phi\left(x_{1}, \ldots, x_{L}\right) \\
& =\frac{1}{\pi^{\frac{D L}{2}}} \int \frac{d^{D} x_{1^{\prime}} \ldots d^{D} x_{L^{\prime}} \Phi\left(x_{1^{\prime}}, \ldots, x_{L^{\prime}}\right)}{\left|x_{11^{\prime}}\right|^{D-2 \omega} \ldots\left|x_{L L^{\prime}}\right|^{D-2 \omega} \times\left|x_{1^{\prime} 2^{\prime}}\right|^{2 \omega} \ldots\left|x_{L^{\prime} 1^{\prime}}\right|^{2 \omega}}
\end{aligned}
$$

schematically presented in Fig. 3. It is easy to see that a power of this operator $\mathcal{H}_{L}^{M}$ generates a fishnet Feynman graph with topology of a cylinder of length $M$ with the circumference $L$. Now, in analogy with the 4D observation of Ref. [12], we notice that this operator can be related to the transfer matrix of the integrable $S O(1, D+1)$ conformal Heisenberg spin chain [24] presented in Fig. 2,

$$
\mathbb{T}(u)=\operatorname{Tr}_{0}\left[\left(R_{01}(u) R_{02}(u) \ldots R_{0 L}(u)\right]\right.
$$

where $u$ is the spectral parameter, and the $R$ matrix acts as an integral operator

$$
\begin{aligned}
& {\left[R_{12} \Phi\right]\left(x_{1}, x_{2}\right)(u)} \\
& =c(u, D, \omega) \int \frac{d^{D} x_{1^{\prime}} d^{D} x_{2^{\prime}} \Phi\left(x_{1^{\prime}}, x_{2^{\prime}}\right)}{\left(x_{12}^{2}\right)^{-u-\frac{D}{4}}\left(x_{21^{\prime}}^{2}\right)^{\frac{D}{4}+u+\omega}\left(x_{12^{\prime}}^{2}\right)^{\frac{3 D}{4}+u-\omega}\left(x_{1^{\prime} 2^{\prime}}^{2}\right)^{-u+\frac{D}{4}}},
\end{aligned}
$$

with the normalization constant

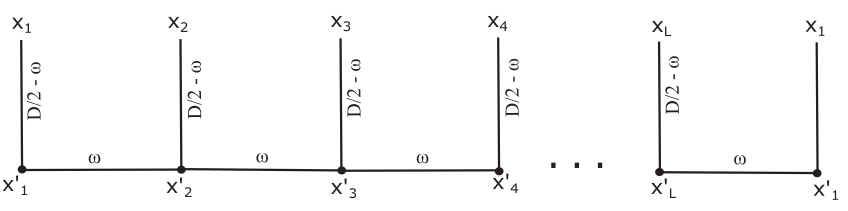

FIG. 3. Graphical representation of the kernel of the graphbuilding operator for generic $D$ and $\omega$. It is obtained by setting $u=-(D / 4)$ in the transfer matrix (8) presented in Fig. 2 so that $x_{j j^{\prime}+1}$-type propagators disappear, while $x_{j^{\prime}+1}-y_{j}$-type propagators are replaced by $\delta^{(D)}\left(x_{j^{\prime}+1}-y_{j}\right)$ factors. After that, integration over the points $y_{j}$ is equivalent to setting $y_{j}=x_{j^{\prime}+1}$.

$$
c(u, D, \omega)=\frac{4^{2 u}}{\pi^{D}} \frac{\Gamma\left(u+\frac{D}{4}+\omega\right) \Gamma\left(u+\frac{3 D}{4}-\omega\right)}{\Gamma\left(-u-\frac{D}{4}+\omega\right) \Gamma\left(-u+\frac{D}{4}-\omega\right)} .
$$

Indeed, in analogy with the 4D case [12], at a particular value of the spectral parameter, this transfer matrix becomes the graph-building operator (7) at any $D$,

$$
\mathcal{H}_{L}=\pi^{-\frac{D L}{2}}\left[\left(4 \pi^{2}\right)^{\frac{D}{2}} \Gamma\left(\frac{D}{2}\right)\right]_{\epsilon \rightarrow 0}^{L} \lim _{\epsilon} \epsilon^{L} \mathbb{T}\left(-\frac{D}{4}+\epsilon\right)
$$

presented in Fig. 3. Thus, this operator is one of the conserved charges of the equivalent spin chain: $\left[\mathbb{T}(u), \mathbb{T}\left(u^{\prime}\right)\right]=\left[\mathbb{T}(u), \mathcal{H}_{L}\right]=0$.

Exact four-point correlation function.-In analogy with the $4 \mathrm{D}$ results of Ref. [16], employing the $D$-dimensional conformal symmetry of the theory (1) and (4), we will compute exactly the four-point correlation function

$$
G=\left\langle O\left(x_{1}, x_{2}\right) \bar{O}\left(x_{3}, x_{4}\right)\right\rangle=\frac{\mathcal{G}(u, v)}{(2 \pi)^{D}\left(x_{12}^{2} x_{34}^{2}\right)^{\frac{D}{4}}},
$$

where the notation is introduced for the operators $O(x, y)=$ $\operatorname{tr}\left[\phi_{1}(x) \phi_{1}(y)\right]$ and $\bar{O}(x, y)=\operatorname{tr}\left[\phi_{1}^{\dagger}(x) \phi_{1}^{\dagger}(y)\right]$. Here, $\mathcal{G}(u, v)$ is a finite function of cross-ratios $u=x_{12}^{2} x_{34}^{2} /\left(x_{13}^{2} x_{24}^{2}\right)$ and $v=x_{14}^{2} x_{23}^{2} /\left(x_{13}^{2} x_{24}^{2}\right)$, invariant under the exchange of points $x_{1} \leftrightarrow x_{2}$ and $x_{3} \leftrightarrow x_{4}$. The OPE expansion leads to the formula

$$
\mathcal{G}(u, v)=\sum_{\Delta} \sum_{S / 2 \in \mathbb{Z}_{+}} C_{\Delta, S}^{2} u^{(\Delta-S) / 2} g_{\Delta, S}(u, v),
$$

where the sums run over operators with scaling dimensions $\Delta$ and even Lorentz spin $S$. Here, $C_{\Delta, S}$ is the corresponding OPE coefficient (structure constant), and $g_{\Delta, S}(u, v)$ is the known $D$-dimensional conformal block [see Eq. (2.9) and Secs. 4 and 5 in Ref. [25]]. If we compute Eq. (11), we will identify the conformal data for the operators emerging in the OPE of $O\left(x_{1}, x_{2}\right)$.

In the planar limit, $G$ is given by the set of fishnet Feynman diagrams presented in Fig. 4. Summing up the corresponding perturbation series, we encounter a geometric progression involving the combination of operators 


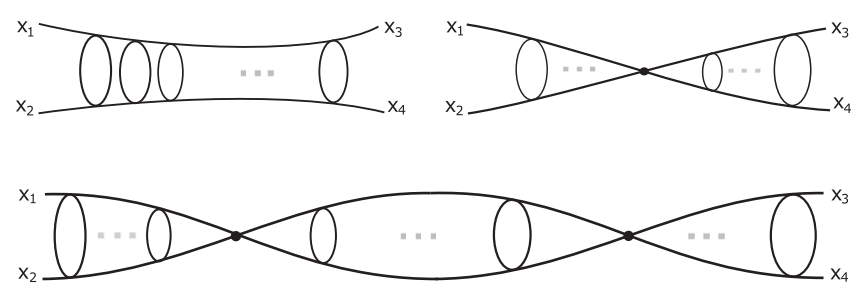

FIG. 4. General fishnet graphs up to $\alpha_{1}^{2}$ order in the expansion of four-point function (13).

$\alpha^{2} \mathcal{V}+\xi^{4} \mathcal{H}_{2}$, where $\alpha^{2}=\alpha_{ \pm}^{2}$ is the double-trace coupling at the fixed point, $\mathcal{V}$ is the operator inserting the double-trace vertex

$$
\mathcal{V} \Phi\left(x_{1}, x_{2}\right)=\frac{2}{\pi^{\frac{D}{2}}} \int \frac{d^{D} x_{1^{\prime}} d^{D} x_{2^{\prime}} \delta^{(D)}\left(x_{1^{\prime} 2^{\prime}}\right) \Phi\left(x_{1^{\prime}}, x_{2^{\prime}}\right)}{\left|x_{11^{\prime}}\right|^{D / 2}\left|x_{22^{\prime}}\right|^{D / 2}}
$$

which is the $D$-dimensional version of Eq. (11) in Ref. [16], and the operator $\mathcal{H}_{2}$ defined by Eq. (7) adds a scalar loop inside the diagram. Hence, we obtain the following representation,

$$
\begin{aligned}
G= & \frac{1}{(2 \pi)^{D}} \int \frac{d^{4} x_{3^{\prime}} d^{4} x_{4^{\prime}}}{\left(x_{33^{\prime}}^{2} x_{44^{\prime}}^{2}\right)^{\frac{D}{4}}}\left\langle x_{1}, x_{2}\left|\frac{1}{1-\alpha^{2} \mathcal{V}-\xi^{4} \mathcal{H}_{2}}\right| x_{3}^{\prime}, x_{4}^{\prime}\right\rangle \\
& +\left(x_{1} \leftrightarrow x_{2}\right),
\end{aligned}
$$

where $x_{i j} \equiv x_{i}-x_{j}[26]$.

Remarkably, the operators $\mathcal{V}$ and $\mathcal{H}_{2}$ commute with the generators of the conformal group, as in the particular 4D case [16]. This fixes the form of their eigenstates

$\Phi_{\Delta, S, n}\left(x_{10}, x_{20}\right)=\frac{1}{\left(x_{12}^{2}\right)^{\frac{D}{4}}}\left(\frac{x_{12}^{2}}{x_{10}^{2} x_{20}^{2}}\right)^{(\Delta-S) / 2}\left(\partial_{0} \ln \frac{x_{20}^{2}}{x_{10}^{2}}\right)^{S}$,

where $\Delta=(D / 2)+2 i \nu$ and $\partial_{0} \equiv\left(\hat{n} \cdot \partial_{x_{0}}\right)$. The state $\Phi_{\Delta, S, n}$ belongs to the principal series of the conformal group and can be represented in the form of a conformal three-point correlation function

$$
C_{\Delta, S} \Phi_{\Delta, S, n}\left(x_{10}, x_{20}\right)=\left\langle\operatorname{tr}\left[\phi_{1}\left(x_{1}\right) \phi_{1}\left(x_{2}\right)\right] O_{\Delta, S, n}\left(x_{0}\right)\right\rangle,
$$

where the operator $O_{\Delta, S, n}\left(x_{0}\right)$ carries the scaling dimension $\Delta$ and Lorentz spin $S$, and $C_{\Delta, S}$ is the three-point structure constant. The states Eq. (14) satisfy the orthogonality condition $[27,28]$

$$
\begin{aligned}
\int \frac{d^{D} x_{1} d^{D} x_{2}}{\left(x_{12}^{2}\right)^{\frac{D}{2}}} \overline{\Phi_{\Delta^{\prime}, S^{\prime}, n^{\prime}}}\left(x_{10^{\prime}}, x_{20^{\prime}}\right) \Phi_{\Delta, S, n}\left(x_{10}, x_{20}\right) \\
=c_{1}(\nu, S) \delta\left(\nu-\nu^{\prime}\right) \delta_{S, S^{\prime}} \delta^{(D)}\left(x_{00^{\prime}}\right)\left(n n^{\prime}\right)^{S} \\
\quad+c_{2}(\nu, S) \delta\left(\nu+\nu^{\prime}\right) \delta_{S, S^{\prime}} Y^{S}\left(x_{00^{\prime}}\right) /\left(x_{00^{\prime}}^{2}\right)^{\frac{D}{2}-S-2 i \nu},
\end{aligned}
$$

where $\Delta^{\prime}=(D / 2)+2 i \nu^{\prime}, Y\left(x_{00^{\prime}}\right)=\left(n \partial_{x_{0}}\right)\left(n^{\prime} \partial_{x_{0^{\prime}}}\right) \ln x_{00^{\prime}}^{2}$, and

$$
\begin{aligned}
c_{1}(\nu, S)= & \frac{2^{S+1} S !|\Gamma(2 i \nu)|^{2}\left(4 \nu^{2}+\left(\frac{D}{2}+S-1\right)^{2}\right)^{-1}}{\pi^{-(3 D / 2+1)}\left|\Gamma\left(\frac{D}{2}-1+2 i \nu\right)\right|^{2} \Gamma\left(\frac{D}{2}+S\right)}, \\
c_{2}(\nu, S)= & \frac{2(-1)^{S} \Gamma^{2}\left(\frac{D}{4}+\frac{S}{2}-i \nu\right)}{\pi^{-(D+1)} \Gamma^{2}\left(\frac{D}{4}+\frac{S}{2}+i \nu\right)} \frac{\Gamma(2 i \nu)}{\Gamma\left(\frac{D}{2}+2 i \nu-1\right)}, \\
& \times \frac{\Gamma\left(\frac{D}{2}+S+2 i \nu-1\right) S !}{\Gamma\left(\frac{D}{2}+S-2 i \nu\right) \Gamma\left(\frac{D}{2}+S\right)} .
\end{aligned}
$$

Calculating the corresponding eigenvalues of the operators $\mathcal{V}$ and $\mathcal{H}$, we find

$$
\begin{aligned}
\mathcal{V} \Phi_{\Delta, S, n}\left(x_{1}, x_{2}\right) & =\delta(\nu) \delta_{S, 0} \Phi_{\Delta, S, n}\left(x_{1}, x_{2}\right), \\
\mathcal{H} \Phi_{\Delta, S, n}\left(x_{1}, x_{2}\right) & =h_{\Delta, S}^{-1} \Phi_{\Delta, S, n}\left(x_{1}, x_{2}\right),
\end{aligned}
$$

where the function $h(\Delta, S)$ is given by Eq. (6). Applying Eqs. (15)-(17), we can expand the correlation function (13) over the basis of states (14). This yields the expansion of $G$ over conformal partial waves defined by the operators $O_{\Delta, S}\left(x_{0}\right)$ in the OPE channel $O\left(x_{1}, x_{2}\right)$,

$$
\mathcal{G}(u, v)=\sum_{S / 2 \in \mathbb{Z}_{+}} \int_{-\infty}^{\infty} d \nu \mu_{\Delta, S} \frac{u^{(\Delta-S) / 2} g_{\Delta, S}(u, v)}{h_{\Delta, S}-\xi^{4}}
$$

where $\Delta=(D / 2)+2 i \nu$, and $\mu_{\Delta, S}=2 \pi^{D} / c_{2}(\nu, S)$ is related to the norm of the state (15). The fact that the dependence on $\alpha^{2}$ disappears from Eq. (18) can be understood as follows. Viewed as a function of $S, \xi^{4} / h_{\Delta, S}$ develops poles at $\nu= \pm i S$ which pinch the integration contour in Eq. (18) for $S \rightarrow 0$. The contribution of the operator $\mathcal{V}$ is needed to make a perturbative expansion of (18) well defined. For finite $\xi^{4}$, these poles provide a vanishing contribution to Eq. (18) but generate a branch-cut $\sqrt{-\xi^{4}}$ singularity of $\mathcal{G}(u, v)$, as in the $4 \mathrm{D}$ case [16].

At small $u$, we close the integration contour in Eq. (18) to the lower half-plane and pick up residues at the poles located at solutions of Eq. (6) and satisfying the unitarity bound $\operatorname{Re} \Delta>S$. The resulting expression for $\mathcal{G}(u, v)$ takes the expected form (12), with the OPE coefficients given by

$$
\begin{aligned}
C_{\Delta, S}^{2}= & \frac{\Gamma\left(\frac{D}{2}+S\right)}{S !} \frac{\Gamma(\Delta-1)}{\Gamma\left(\Delta-\frac{D}{2}\right)} \operatorname{Res}\left(\frac{d \Delta}{h_{\Delta, S}-\xi^{4}}\right) \\
& \times \frac{\Gamma(S-\Delta+D) \Gamma^{2}\left(\frac{1}{2}(S+\Delta)\right)}{\Gamma^{2}\left(\frac{1}{2}(S-\Delta+D)\right) \Gamma(S+\Delta-1)},
\end{aligned}
$$

where the residue is computed with respect to the appropriate solution of Eq. (6) for each relevant operator. For instance, we can consider $\operatorname{tr}\left(\phi_{1}^{2}\right)^{\dagger}$, which is exchanged for any even $D$; then the perturbative expansion of Eq. (19) is 
$C_{\mathrm{tr} \phi^{2}}^{2}=2+\frac{4 i \xi}{\Gamma\left(\frac{D}{2}\right)}\left[2 \psi^{(0)}\left(\frac{D}{4}\right)-\psi^{(0)}\left(\frac{D}{2}\right)+\gamma_{E}\right]+O\left(\xi^{2}\right)$.

The relations (6) and (19) define exact conformal data of operators propagating in the OPE channel $x_{1} \rightarrow x_{2}$.

Finally, we discuss an interesting $D \rightarrow \infty$ limit of the theory. We should then rescale the coupling $\xi=\xi_{\infty} \sqrt{\Gamma(D / 2)}$, where $\xi_{\infty}$ is fixed. The anomalous dimension $\gamma_{\infty}$ of $\operatorname{tr}\left(\phi_{1}^{2}\right)$ has finite limit since for $S=0$ in Eq. (6) it is given by

$$
-\gamma_{\infty} \sin \left(\frac{\pi \gamma_{\infty}}{2}\right)=2 \pi \xi_{\infty}^{4}
$$

while $\gamma_{\infty}$ vanishes for $\operatorname{tr}\left(\phi_{1} \partial_{+}^{S} \phi_{1}\right)$ operators. Concerning the expansion (12), the number of exchanged operators becomes a countable infinity diverging linearly in $D$. Finally, the OPE structure constant (20) for $\operatorname{tr}\left(\phi_{1}^{2}\right)$ trivially reduces to its bare value in this limit.

Conclusions. - We showed that the strongly $\gamma$-deformed $\mathcal{N}=4$ SYM theory proposed in Ref. [5] is just the fourdimensional representative of a wider, $D$-dimensional family of theories of two complex scalar fields obtained by modifying the propagators of fields in a $D$-dependent way. Similar to the 4D case [16], they turn out to be conformal and integrable at any $D$, at least in the planar limit, if we add to the action certain double-trace terms with specific couplings. The conformality of our theory at finite $N_{c}$ remains an open question, though it is quite plausible that the planar conformal point simply shifts to some other complex values of couplings. There are two such complex conjugate values of these couplings, and we computed them perturbatively up to two loops. The integrability is explicit due to the domination of sufficiently large orders of perturbation theory by the fishnet Feynman diagrams. The cylindric fishnet graphs related to the renormalization of vacuum $\operatorname{tr}\left(\phi_{1}^{L}\right)$ operators can be created by multiple application of a graph-building operator which appears to be an integral of motion of the integrable conformal $S O(1, D+1)$ spin chain. We also generalized the biscalar model to a CFT with different propagators for the fields $\phi_{1}$ and $\phi_{2}$, leading to nonisotropic fishnet Feynman graphs. The underlying graph-building operator has representations with different conformal spins in two directions on the fishnet graph. In the 2D case, the fishnet graphs are described by the same $S L(2, \mathbb{C})$ chain as used for the dynamics of generalized Lipatov's Reggeized gluons [29] but with different value of spin, $s=1 / 4$ in isotropic case, instead of the Balitski-Fadin-Kuraev-Lipatov (BFKL) Reggeized gluon spin $s=0$. This spin chain extensively studied in the literature [30-35] is restored in the singular limit $\omega \rightarrow 0$ of our biscalar model (1). In the spirit of Ref. [16], we computed here the exact four-point correlator at any $D$ as an expansion into conformal blocks with explicit OPE coefficients and dimensions of exchange operators in one of the channels. In the 1D case, our results were similar to the scalar version of conformal Sachdev-Ye-Kitaev (cSYK) fermionic theory [36] at $q=4$. For even $D$, we found a finite $D$-dependent number of local exchange operators at a given spin and dimension. It would be very interesting to compute some of the discussed quantities (dimensions, structure constants) in the next $1 / N_{c}^{2}$ approximation, similar to Refs. [37-39], if the conformality of the theory holds at any $N_{c}$. The explicit form of these operators can be obtained by the analysis of the mixing matrix for their quantum multiplets $[12,20]$. This becomes more complicated as the dimension grows due to growing rank of the multiplets and the number of transitions, together with log-CFT effects which arise starting from $4 \mathrm{D}$, due to the chirality.

Although the Lagrangian (1) of our theory is nonlocal at general $D$ (apart from the sequence $D \in 4 \mathbb{N}$ in the isotropic case), it does not prevent the existence of "normal" OPE data in this theory, which is more important for the physical interpretation of this CFT. Moreover, it would be interesting to generalize to any $D$ the results for fishnet graphs of the type considered in Ref. [40] and to the correlation functions for operators involving more than two scalars. Finally, an important question remains, as in $4 \mathrm{D}$, whether these theories have any string duals at any $D$, according to the original proposal of 't Hooft [41].

We thank B. Basso, J. Caetano, S. Derkachov, N. Gromov, G. Korchemsky, and F. Levkovich-Maslyuk for numerous useful discussions. The work of V. K. was supported by the European Research Council (Programme "Ideas" Grant No. ERC-2012-AdG 320769 AdS-CFT-solvable). The work of E. O. is supported by the German Science Foundation (DFG) under the Collaborative Research Center (SFB) 676 Particles, Strings and the Early Universe and the Research Training Group 1670.

[1] P. Di Francesco, P. Mathieu, and D. Senechal, Conformal Field Theory, Graduate Texts in Contemporary Physics, (Springer-Verlag, New York, 1997).

[2] T. Banks and A. Zaks, Nucl. Phys. B196, 189 (1982).

[3] N. Beisert et al., Lett. Math. Phys. 99, 3 (2012).

[4] The integrability of such theories emerges due to their duality to string sigma models on specific cosets having an infinite number of quantum conservation lows for their world-sheet dynamics or due to the analogy between the planar Feynman diagram technique and integrable $(1+1) D$ quantum spin chains [3].

[5] O. Gürdogan and V. Kazakov, Phys. Rev. Lett. 117, 201602 (2016).

[6] N. Gromov, V. Kazakov, S. Leurent, and D. Volin, Phys. Rev. Lett. 112, 011602 (2014).

[7] N. Gromov, V. Kazakov, and P. Vieira, Phys. Rev. Lett. 103, 131601 (2009). 
[8] V. Kazakov, S. Leurent, and D. Volin, J. High Energy Phys. 12 (2016) 044.

[9] J. Caetano, O. Gurdogan, and V. Kazakov, J. High Energy Phys. 03 (2018) 077.

[10] A. B. Zamolodchikov, Phys. Lett. 97B, 63 (1980).

[11] In the rest of the Letter, we will use its Euclidean version $S O(1, D+1)$ instead of the Minkowskian $S O(2, D)$.

[12] N. Gromov, V. Kazakov, G. Korchemsky, S. Negro, and G. Sizov, J. High Energy Phys. 01 (2018) 95.

[13] J. Fokken, C. Sieg, and M. Wilhelm, J. Phys. A 47, 455401 (2014).

[14] J. Fokken, C. Sieg, and M. Wilhelm, J. High Energy Phys. 09 (2014) 78.

[15] C. Sieg and M. Wilhelm, Phys. Lett. B 756, 118 (2016).

[16] D. Grabner, N. Gromov, V. Kazakov, and G. Korchemsky, Phys. Rev. Lett. 120, 111601 (2018).

[17] This term can be computed at any $D$ by means of integration by parts and Mellin-Barnes transformation, according to Ref. [18].

[18] A. G. Grozin, Int. J. Mod. Phys. A 27, 1230018 (2012).

[19] Here and in the following, we adopt the notation $\psi^{(k-1)}(x)=$ $\left[\left(d^{k}\right) /\left(d x^{k}\right)\right]+\log \Gamma(x)$ for polygamma functions.

[20] J. Caetano (unpublished).

[21] V. Gurarie, Nucl. Phys. B410, 535 (1993).

[22] M. Hogervorst, M. Paulos, and A. Vichi, J. High Energy Phys. 10 (2017) 201.

[23] This terminology for the graphs of regular square lattice shape was introduced by Sakita and Virasoro in 1970.

[24] D. Chicherin, S. Derkachov, and A. P. Isaev, J. High Energy Phys. 04 (2013) 020.
[25] F. A. Dolan and H. Osborn, arXiv:1108.6194.

[26] The operators $\mathcal{V}$ and $\mathcal{H}$ are not well defined separately; e.g., for an arbitrary $\Phi\left(x_{i}\right)$, the expressions for $\alpha^{4} \mathcal{V}^{2} \Phi\left(x_{i}\right)$ and $\xi^{4} \mathcal{H} \Phi\left(x_{i}\right)$ are given by divergent integrals. However, at the fixed point, their sum is finite by virtue of conformal symmetry.

[27] V. K. Dobrev et al., Lect. Notes Phys. 63, 1 (1977).

[28] E. S. Fradkin and M. Y. Palchik, Phys. Rep. 44, 249 (1978).

[29] L. N. Lipatov, JETP Lett. 59, 596 (1994).

[30] G. P. Korchemsky, Nucl. Phys. B550, 397 (1999).

[31] S. E. Derkachov, G. P. Korchemsky, and A. N. Manashov, Nucl. Phys. B617, 375 (2001).

[32] H. J. de Vega and L. N. Lipatov, Phys. Rev. D 64, 114019 (2001).

[33] I. Balitsky, V. Kazakov, and E. Sobko, arXiv:1310.3752.

[34] I. Balitsky, V. Kazakov, and E. Sobko, arXiv:1511.03625.

[35] I. Balitsky, V. Kazakov, and E. Sobko, Phys. Rev. D 93, 061701 (2016).

[36] D. J. Gross and V. Rosenhaus, J. High Energy Phys. 12 (2017) 148.

[37] B. Eden, Y. Jiang, D. le Plat, and A. Sfondrini, J. High Energy Phys. 02 (2018) 170.

[38] T. Bargheer, J. Caetano, T. Fleury, S. Komatsu, and P. Vieira, arXiv:1711.05326.

[39] R. Ben-Israel, A. G. Tumanov, and A. Sever, J. High Energy Phys. 08 (2018) 122.

[40] B. Basso and L. J. Dixon, Phys. Rev. Lett. 119, 071601 (2017).

[41] G. 't Hooft, Nucl. Phys. B72, 461 (1974). 